\title{
Efecto del tratamiento sobre el descuento temporal y probabilístico en participantes con trastorno por uso de crack
}

\author{
Diana Mejía', Silvia Morales-Chainé', Javier Nieto' \\ ' Facultad de Psicología. Universidad Nacional Autónoma de México, México
}

\section{RESUMEN}

Introducción: diversos estudios han mostrado que individuos con dependencia de drogas psicoactivas muestran un descuento temporal pronunciado de recompensas monetarias en contraste con no consumidores. Este hallazgo resulta de utilidad clínica como una característica conductual susceptible a modificación por tratamientos psicológicos. Objetivo: evaluar modificaciones pre-post en descuento temporal y probabilístico, sintomatología somática, ansiedad, depresión, psicosis y autoeficacia, en participantes con trastorno por uso de crack severo que recibieron una de dos modalidades de tratamiento psicológico y en un grupo control sin consumo. Método: se trabajó con 92 participantes, 31 en cada uno de los tratamientos y 30 en el grupo control. Se evaluó a los tres grupos de manera pre-post; el post se llevó a cabo después de 40 días para los tres grupos, tiempo durante el cual recibían alguna de las dos modalidades de atención, a excepción del grupo control, al cual se le dio cita para la evaluación post. Resultados: las mediciones pre-post de descuento temporal y probabilístico no cambiaron en ninguno de los tres grupos. En contraste, ambas modalidades de tratamiento incrementaron la autoeficacia y disminuyeron síntomas somáticos como ansiedad, depresión y psicoticismo. Discusión y conclusiones: se demostró la estabilidad de las mediciones de descuento ante el tratamiento psicológico; otras variables sí fueron susceptibles de modificación debido al tratamiento, por lo que la utilidad clínica del descuento, como una variable que indique el éxito de la intervención, debe seguir explorándose en futuras investigaciones.

Palabras clave: descuento temporal, descuento probabilístico, cocaína, tratamiento, impulsividad y búsqueda de riesgo.
ABSTRACT
Introduction: several studies have shown that individuals with dependence on psychoactive drugs show a steeply delay discounting with monetary rewards in contrast to non-consumers. This finding is clinically useful as a behavioral trait likely to be modified by psychological treatments. Objective: this study evaluated changes pre-post measures of delay and probability discounting, somatic symptoms, anxiety, depression, psychosis and self-efficacy in participants with crack use disorder severe who received one of two types of psychological treatment. Method: we worked with ninety two participants, 31 for each treatment and 30 in the control group. We evaluated the three groups so pre-post, the post was held after 40 days for the three groups, and during that time the cocaine users received one of the two forms of treatments, except control who only received post evaluation. Results: the results showed that the pre-post evaluation with probability and delay discounting procedures did not change in any of the three groups. Suggesting that the discounting is not sensitive to identify short-term changes as result from psychological interventions. In contrast both treatment modalities increased self-efficacy, and reduced symptoms of somatization, anxiety, depression and psychosis. Not a main effect for treatment type was found. Discussion and conclusions: this study showed the stability of discounting measures with psychological treatment. While other variables they were subject to change due to the treatment, so the clinical utility of the discounting as a variable indicating the success of the intervention should be further explored in future research.

Key words: delay discounting, probability discounting, cocaine, treatment, impulsivity and risk taking.

\footnotetext{
Autor de correspondencia:

Diana Mejía. Cerro de Acasulco núm. 18, col. Oxtopulco Universidad, del. Coyoacán, C.P. 04318, Ciudad de México.

Tel. +52 (55) 5658 3911. Correo electrónico: d.psicologia@hotmail.com

Recibido: 11 de abril de 2016.

Aceptado: 18 de mayo de 2016.

DOI: 10.28931/riiad.2016.1.02
} 


\section{INTRODUCCIÓN}

En los últimos 15 años ha surgido una serie de estudios que demuestran, de manera contradictoria, cambios y estabilidad en las mediciones de descuento en usuarios de drogas, esto con el objetivo primordial de disminuir la tendencia de elección, denominada impaciente, de recompensas inmediatas de menor magnitud que caracteriza a los usuarios de drogas. Se ha demostrado que las tasas de descuento temporal y probabilístico son estables a tres meses en participantes sin uso de drogas que no recibían atención psicológica (Ohmura, Takahashi, Kitamura, \& Wehr, 2006). Este hallazgo podría suponer que aquellos usuarios de drogas que acuden a recibir algún tipo de intervención psicológica modificarán su descuento, en contraste con los que no reciban la intervención. Se ha demostrado que las tasas de descuento temporal en ganancias de dinero son estables después del tratamiento cognitivo-conductual, el entrenamiento en atención plena y el manejo de contingencias (MC) en usuarios de alcohol (Dennhardt, Yurasek, \& Murphy, 2015; De Wilde, Bechara, Sabbe, Hulstijn, \& Dom, 2013; Jones, 2013), tabaco (López, 2014; López-Torrecillas, Perales, Nieto-Ruiz, \& Verdejo-García, 2014; Secades-Villa, Weidberg, García-Rodríguez, Fernández-Hermida, \& Yoon, 2014; Weidberg, García-Rodríguez, Yoon, \& Secades-Villa, 2015) y otras drogas ilegales (Weidel, 2013). En contraste con estos estudios, Landes y colaboradores (2012) encontraron en usuarios con dependencia de opioides que $48 \%$ de la muestra no cambiaba sus tasas de descuento, 39\% mostró un decremento de su tasa y $12 \%$ la incrementó, sin interacción con el tipo de tratamiento recibido. Pocos estudios han documentado la existencia de diferencias en las tasas de descuento entre los participantes que logran la abstinencia y los que recaen. Se ha encontrado un mayor descuento temporal en ganancias monetarias entre los participantes que recaen, en comparación con los que mantienen la abstinencia en situaciones experimentales y en escenarios reales de tratamiento (Dallery \& Raiff, 2007; López-Torrecillas et al., 2014).

En contraste, se ha demostrado que las tasas de descuento temporal de ganancias monetarias disminuyen con manejo de contingencia (MC) en usuarios de tabaco (Yi et al., 2008), alcohol y cocaína (Black \& Rosen, 2011). Aunque pareciera que el tratamiento con $\mathrm{MC}$ es consistente en la modificación de las tasas de descuento, Peters y colaboradores (2013) demostraron que éstas disminuyeron en usuarios de mariguana que no reciben MC; en tanto que en consumidores de tabaco presentaron un decremento únicamente en mujeres (Weidberg, Landes, García-Rodríguez, Yoon, \& Secades-Villa, 2015).
En contraste con el tratamiento de MC, se ha demostrado que el entrenamiento en memoria de trabajo decrementó las tasas de descuento temporal de ganancias monetarias en usuarios de estimulantes (Bickel, Yi, Landes, Hill, \& Baxter, 2011). Otra intervención que resultó ser efectiva en la modificación de las tasas de descuento es la Acceptance-Based Procedure, la cual demostró disminución del descuento en estudiantes sin trastorno por consumo de drogas (Morrison, Madden, Odum, Friedel, \& Twohig, 2014).

La evidencia del valor predictivo del descuento en el tiempo de abstinencia o la recaída en consumidores de drogas después de tratamiento es contradictoria, pues algunos estudios han mostrado la asociación en usuarios de tabaco (Krishnan-Sarin et al., 2007; MacKillop \& Kahler, 2009; Sheffer et al., 2012), mariguana (Stanger et al., 2012), cocaína (Washio et al., 2011) y otras drogas (Stevens, Verdejo-García, Roeyers, Goudriaan, \& Vanderplasschen, 2015); sin embargo, otras investigaciones han señalado que no es un predictor del tiempo de abstinencia en usuarios de tabaco (López-Torrecillas, Nieto-Ruiz et al., 2014), alcohol (Jones, 2013), mariguana (Peters et al., 2013) y opioides (Passetti et al., 2011).

Las anteriores investigaciones han utilizado procedimientos con descuento temporal de ganancias demoradas. Sólo una ha evaluado descuento temporal y probabilístico utilizando usuarios de drogas en mediciones pre-post (Yi \& Landes, 2012); en dicho estudio, los investigadores demostraron que después de 24 horas de abstinencia, las tasas de descuento temporal de ganancias y pérdidas monetarias incrementaban en fumadores, mientras que el descuento probabilístico de ganancias y pérdidas monetarias no se modificó. Los hallazgos sugieren que las modificaciones del descuento temporal se pueden relacionar tanto con el síndrome de abstinencia como con un tratamiento relacionado con la intención de dejar el consumo de drogas.

Todavía existe controversia entre diversos estudios que evalúan si las mediciones de descuento se modifican o son estables, por lo tanto es necesario analizar si, después del tratamiento, existen modificaciones en el mismo, en mediciones con variaciones de diferentes tipos de tareas y a través de la utilización de procedimientos temporales y probabilísticos. Todavía se desconoce la utilidad clínica del descuento para predecir recaída y abandono del tratamiento.

En escenarios clínicos, la cocaína representa la tercera droga con más demanda de tratamiento (Marín-Navarrete et al., 2014); la evidencia sustenta que los usuarios de esta sustancia descuentan más pronunciadamente que los grupos control (Coffey, Gudleski, Saladin, \& Brady, 2003; García-Rodríguez, Secades-Villa, Weidberg, \& Yoon, 2013; Heil, Johnson, Higgins, \& Bic- 
kel, 2006). Se ha demostrado, asimismo, que no existe diferencia en el grado de descuento entre policonsumidores de cocaína y monoconsumidores de esta misma droga (García-Rodríguez et al., 2013; Mejía, Morales, \& Nieto, 2015), por lo que resulta necesario llevar a cabo investigación con usuarios de cocaína, sobre todo entre quienes la consumen fumada.

El presente estudio tuvo como hipótesis central la existencia de diferencias entre un entrenamiento cognitivo-conductual y un tratamiento usual, en tasas de descuento, autoeficacia y sintomatología de abstinencia, en una muestra de personas con trastorno por uso de crack en tratamiento residencial.

\section{MÉTODO}

\section{Diseño}

En el presente estudio se planteó un diseño pre-post con dos observaciones, una realizada antes del tratamiento y otra, seis semanas después, en participantes con trastorno por uso de crack equiparado en variables demográficas. Para evaluar la superioridad del entrenamiento cognitivo-conductual frente al tratamiento usual en las variables de interés, se agregó un tercer grupo, cuya medición de descuento permaneciera estable en el tiempo y sirviera como comparación (Kazdin, 2011).

\section{Sedes}

La muestra se obtuvo en dos centros de atención residenciales en la Ciudad de México. En ambos se brinda tratamiento por profesionales de la salud a usuarios con trastorno por uso de drogas, así como atención psicológica, psiquiátrica, médica y de trabajo social.

\section{Participantes}

Los participantes cumplieron los siguientes criterios de inclusión: saber leer y escribir; diagnóstico de trastorno por uso de crack definido con un puntaje > 27 para cocaína en el test de tamizaje de alcohol, tabaco y uso de drogas (ASSIST) (Linage \& Lucio, 2013; WHO ASSIST Working Group, 2002) y un puntaje > 11 en el Cuestionario de Abuso de Drogas (CAD-20) (Skinner, 1982; Villalobos-Gallegos, Pérez-López, Mendoza-Hassey, Graue-Moreno, \& Marín-Navarrete, 2015); asimismo, se les solicitó aceptar participar voluntariamente en la investigación.

Los criterios de exclusión fueron: participantes que cursaron con episodio psicótico o maniaco actual evaluado por la Mini Entrevista Neuropsiquiátrica Internacional (Sheehan, Lecrubier, \& Sheehan, 1998); deterioro cognitivo definido por un puntaje $<6$ en las subpruebas de retención de dígitos y símbolos y dígitos del WAIS (Tulsky \& Zhu, 2003); puntuar >27 en el ASSIST para cualquier otra sustancia diferente a la cocaína.

\section{Instrumentos/Variables}

Symptom Check List 90 (SCL- 90): mediante 90 reactivos, evalúa el grado de distrés psicológico que experimenta el sujeto durante el período comprendido entre el día de la evolución y una semana anterior a la aplicación (Cruz, López, Blas, González, \& Chávez, 2004). El valor del alfa de Cronbach de consistencia interna para siete de las nueve dimensiones, así como el ISG, fue mayor que .7 , en tanto que las restantes obtuvieron puntajes mayores que .66. Se utilizaron para análisis las siguientes subescalas del instrumento: somatización, depresión, ansiedad, psicoticismo e índice de severidad global. Se eligieron estas subescalas de acuerdo con el cuadro de síntomas que el DSM-5 (American Psychiatric Association, 2013) asocia con sintomatología derivada de la intoxicación y síndrome de abstinencia por uso de estimulantes mayores como la cocaína. En cada subescala se promedian los ítems que las componen, los cuales van, en escala Likert, de 0 a 4.

Cuestionario de Confianza Situacional (CCS): permite conocer el nivel de autoeficacia de los usuarios para resistir el consumo ante ocho categorías situacionales. Para su adaptación a población adolescente se aplicó el instrumento a una muestra de 150 jóvenes, de entre 14 y 19 años de edad, usuarios de alcohol y otras drogas; la consistencia interna del instrumento fue de .97 (Echeverría \& Ayala, 1977). Este instrumento presenta una escala de 0 a 100, para indicar el porcentaje de autoeficacia percibida ante ocho situaciones de riesgo asociadas con el consumo de drogas.

\section{Descuento temporal y probabilístico}

El presente estudio utilizó el cálculo de área bajo la curva (AuC) (Myerson, Green, \& Warusawitharana, 2001) de descuento temporal y probabilístico para identificar el grado de devaluación psicológica de los participantes. Esta medida consiste en el cálculo de la extensión de área debajo de los puntos de indiferencia, donde los valores cercanos a 1 indican un descuento poco pronunciado, mientras que uno pronunciado se indica por valores cercanos a cero.

Para cada participante se realizó la programación de 10 tareas, de las cuales se obtuvo el cálculo de 10 AuC (bebida temporal y probabilística; actividad de tiempo libre temporal y probabilística; ganancia \$200 temporal y probabilística; ganancia de $\$ 3000$ temporal y probabilística; pérdida de $\$ 1500$ temporal y probabilística). 
Para la programación de las tareas computacionales se utilizó el cuestionario de bebida, cuyo fin es determinar la bebida no alcohólica preferida del participante. Consistió en seis categorías de tipos de bebidas de 350 $\mathrm{ml}$ en promedio, ejemplo: lata de refresco, jugo enlatado, botella de agua de sabor, botella de agua mineral, botella de té, botella de capuchino frío y vaso de café o capuchino (Manwaring, Green, Myerson, Strube, \& Wilfley, 2011). Se utilizó también la lista de actividades de tiempo libre preferidas, basada en las actividades de tiempo libre utilizadas en los estudios de Epstein y Roiemmich (2001) y Manwaring y colaboradores (2011); algunos ejemplos de las actividades son ver televisión, hacer deporte, ir al teatro y al cine.

Se utilizaron tres computadoras equipadas con el programa de ajuste de la cantidad inmediata, a partir de los procedimientos utilizados por Holt, Green y Myerson (2012).

\section{Procedimiento}

Los participantes fueron reclutados dentro de dos centros de tratamiento residencial entre agosto de 2013 y agosto de 2015. En ambos se destinó una sesión para la administración de las pruebas psicológicas definidas en la sección de instrumentos, pruebas antidoping y pruebas de tareas de elección. Si el usuario no cumplía con los criterios de inclusión, se continuaba con el proceso de evaluación establecido por el centro de atención.

Para determinar si el participante cumplía con los criterios de inclusión, se le administraron las siguientes evaluaciones para saber si era candidato al estudio: ficha demográfica, test ASSIST, CAD-20, subpruebas de retención de dígitos y símbolos y dígitos de las Escalas WAIS, entrevista MINI. Una vez que se determinaba que los participantes cumplían con los criterios de inclusión, se explicaba el consentimiento informado, se asignaba un folio y se aplicaban los siguientes instrumentos: cuestionario de la bebida de preferencia, lista de actividades de tiempo libre, SCL-90 y CCS.

La mitad de los participantes de cada grupo de la variable tipo de droga fue asignada de acuerdo con su folio de participación: para los participantes con folio non, el orden de aplicación fue el paquete de tareas temporales, seguido por el de tareas de probabilidad; para los participantes con folio par, el orden de aplicación fue el paquete de tareas de probabilidad, seguido por el de temporalidad. El orden de la aplicación de cada tipo de tarea fue aleatorio y el orden de demoras o probabilidades fue aleatorio para cada tarea. Se utilizaron cinco demoras (una semana, un mes, seis meses, un año y tres años) y cinco probabilidades (90\%, 75\%, $50 \%, 25 \%$ y $10 \%$ ). Demoras y probabilidades retomadas de Manwaring y colaboradores (2011).
Las tareas de elección se llevaron a cabo en un programa de computadora en el que se utilizó JAVA; en él se empleó el procedimiento de ajuste de la cantidad inmediata, el cual consistió en que para cada cantidad de recompensa, el participante realizaba cinco ensayos de elecciones para las tareas de ganancia de dinero 200, 3000 y pérdida de 1500, y cuatro ensayos para las tareas de consumible líquido y tiempo libre. Estos ensayos se realizaban para cada demora establecida. La cantidad de la recompensa inmediata era la mitad de la cantidad de la de mayor magnitud demorada. Por ejemplo, si la cantidad de la recompensa demorada es de $\$ 100$ pesos en seis meses, entonces en el primer ensayo se le pedía que eligiera entre la recompensa inmediata de $\$ 50$ pesos o $\$ 100$ pesos en seis meses. Para las elecciones subsecuentes, el incremento o decremento en la recompensa de entrega inmediata fue la mitad del ajuste anterior. Si el participante elegía la recompensa inmediata para el siguiente ensayo, la cantidad de esta recompensa inmediata decrecía; por otro lado, si escogía la recompensa demorada para el siguiente ensayo, la cantidad de la recompensa inmediata incrementaba. Este procedimiento se repitió hasta que el participante realizó los cinco o cuatro ensayos de elecciones establecidos; el valor que el participante obtendría en la cantidad variable para un sexto o quinto ensayo era el punto de indiferencia para esa demora. Un procedimiento de ajuste de la cantidad inmediata análogo se utilizó para estimar el valor subjetivo de las recompensas probabilísticas; se consideró el mismo número de ensayos de acuerdo con el tipo de resultado (Holt et al., 2012).

Para las tareas de pérdida monetaria de $\$ 1500$, tanto en procedimientos temporales como probabilísticos, los incrementos y decrementos eran opuestos a las tareas de ganancia; por tanto, elegir el pago inmediato generaba que en el siguiente ensayo la cantidad del resultado inmediato incrementara, mientras que escoger el pago demorado para el siguiente ensayo propiciaba que la cantidad del resultado inmediato disminuyera.

En cada tarea computarizada (demora y probabilidad), las cantidades de la recompensa o resultado demorado y probable se evaluaron de la siguiente manera: dos cantidades de recompensa monetaria ( $\$ 200$ y $\$ 3000$ ) y una cantidad de pérdida (\$1500). En la tarea de bebida de preferencia se evaluó con una cantidad de 40 unidades. Para la recompensa de actividad de tiempo libre, la cantidad era de 100 minutos.

El espacio de evaluación en ambos centros de tratamiento fue un cuarto de $2 \times 2$ metros, con adecuada iluminación y ventilación, con un escritorio y dos sillas. A los participantes se les proporcionó instrucción verbal y escrita para indicar que el objetivo del estudio fue examinar decisiones en situaciones de recompensas 
hipotéticas: cantidades de dinero, una bebida seleccionada por el participante y una actividad de tiempo libre preferida.

Una vez que se evaluó a los participantes consumidores de drogas, se obtuvieron las características demográficas que permitieron conformar un grupo control equivalente pero sin consumo; las variables utilizadas para conformar el grupo control fueron edad y nivel socioeconómico.

En los controles se omitió la aplicación del cuestionario de confianza situacional; sin embargo, se aplicaron las demás pruebas mediante el procedimiento antes descrito.

De entre los participantes que se encontraban en tratamiento residencial, un grupo recibió el tratamiento usual del centro y un tratamiento cognitivo-conductual en autocontrol grupal. La elección del tipo de tratamiento asignado a cada participante dependió del tiempo, ya que durante el primer año del estudio se monitoreó el tratamiento usual y en el segundo se implementó el tratamiento grupal en autocontrol debido al espacio y las actividades asignadas por ambos centros residenciales. Se buscó la equivalencia en los grupos de tratamiento de acuerdo con la edad (entre 18 y 51 años), los años de educación (11 y 16 años) y los puntajes obtenidos en el ASSIST y el CAD-20.

\section{Sección de tratamiento}

El tratamiento usual constaba de sesiones individuales realizadas una vez por semana, con enfoque ecléctico, por parte del personal de psicología; charlas grupales psicoeducativas impartidas diariamente por el profesional médico, acerca de la importancia de una dieta adecuada y del apego a los medicamentos; y pláticas de búsqueda de empleo y proyecto de vida, impartidas por el personal de trabajo social. Además, incluía la medicación psiquiátrica administrada durante seis semanas con neuromoduladores, antipsicóticos y antidepresivos.

Al grupo con tratamiento cognitivo-conductual en autocontrol se le brindó el siguiente tratamiento, además de todas las actividades anteriormente descritas en el grupo con tratamiento usual. El tratamiento cognitivo-conductual en autocontrol grupal es derivado del tratamiento denominado "Programa de Satisfactores Cotidianos (PSC)" (Barragán, Flores, Medina-Mora, \& Ayala, 2007). Se utilizó la adaptación grupal del tratamiento PSC a seis componentes (Mejía, 2012), el cual consistió en una sesión por semana con una duración de dos horas cada una. Las habilidades impartidas fueron las siguientes: análisis funcional de la conducta de consumo, muestra de abstinencia, control del deseo de consumo, rehusarse al consumo, metas de vida, solución de problemas, control de ansiedad, control de depresión, control de enojo, habilidades de comunicación, habilidades sociales recreativas y prevención de recaídas.

La última semana en el tratamiento residencial se llevó a cabo la evaluación postratamiento, en la que se aplicaron las escalas SCL-90, CCS y tareas de elección, con el mismo procedimiento anteriormente señalado. Con los participantes del grupo control sólo se trabajó de manera pre y post después de seis semanas. Ninguno de los participantes recibió durante este período tratamiento psicológico o psiquiátrico.

Después de tres meses de haber finalizado el tratamiento, se realizó un seguimiento telefónico con los usuarios de drogas para evaluar su tiempo en abstinencia; la información recabada se verificó de manera telefónica con sus familiares.

\section{Análisis de resultados}

Se calcularon los valores individuales del área bajo de la curva para las 10 tareas (AuC) (Myerson et al., 2001); se obtuvieron los puntajes promedios individuales prepost en cinco subescalas del SCL-90. También se calculó el puntaje promedio de las ocho situaciones de riesgo pre-post del cuestionario CCS, para evaluar los efectos de interacción tiempo y factor participantes (tratamiento usual, tratamiento autocontrol y control).

Se realizó un modelo de ANOVA para medidas repetidas pre-post de AuC con los factores participantes: tipo de tareas (ganancias: bebida de preferencia, actividad libre, $\$ 200$, \$3000 y pérdida \$1500); y tipo de procedimientos (temporal y probabilístico). Se efectuaron comparaciones planeadas para evaluar diferencias en tipo de participantes y cambios por tipo de tareas.

Posteriormente, se realizó un análisis de varianza para medidas repetidas; éste se realizó de manera separada para las siguientes variables dependientes: cinco subescalas del SCL-90 y CCS. Además, se consideró el factor tipo de participante en cada uno de los análisis de las variables dependientes.

De los 62 participantes que terminaron su tratamiento, 20 permanecieron en abstinencia, 25 recayeron y se perdió el seguimiento de 17. Considerando el tipo de tratamiento, se realizó un análisis de Chi-cuadrada para evaluar si la recaída y el abandono se asociaban al tipo de tratamiento recibido. Después, se realizó una $t$ de Student para muestras independientes, a fin de comparar a los participantes que mantenían la abstinencia con aquellos que recaían a los tres meses de seguimiento; se consideraron los valores del post test de las variables AuC, subescalas SCL-90 y CCS. La recaída a tres meses se entendió como regresar al patrón de consumo previo al tratamiento. 
Tras esto, se realizó una $t$ de Student para muestras independientes, con la intención de comparar participantes que terminaron tratamiento con los que no lo lograron; se contrastaron los valores pre de las variables AuC, subescalas SCL-90 y CCS.

\section{Consideraciones éticas}

Todos los participantes firmaron un consentimiento informado de participación en el estudio, en el que se explicaron los objetivos de la investigación y los riesgos y beneficios; asimismo, se estableció la posibilidad de dejar de participar en el estudio en cualquier momento.

\section{RESULTADOS}

Se analizaron los datos de 92 hombres de entre 18 y 51 años, 62 usuarios de cocaína en piedra y 30 controles sin consumo de drogas. En los grupos se presentaron diferencias significativas, con respecto a sus características en edad y nivel educativo (ver Tabla 1).

La prueba de Levene no fue significativa, por lo que supone homogeneidad de varianza a pesar del desbalance en la $\mathrm{N}$ de los participantes. No se encontró interacción significativa entre los cuatro factores definidos en análisis $[F(8,890)=.49, p=.85]$; tampoco interacción entre los factores pre-post, procedimiento y tareas $[F(4,890)=.27, p=.89]$; no hubo interacción entre los factores pre-post, participantes y tareas $[F(8,890)=$ 1.34, $p=.21]$; no hubo interacción entre los factores pre-post, participantes y procedimientos $[F(2,890)=$ .24, $p=.78]$; no hubo interacción entre los factores pre-post y participantes $[F(2,890)=.52, p=.59]$; no hubo interacción entre los factores pre-post y procedimientos $[F(1,890)=.07, p=.79]$. En contraste, se encontró interacción entre los factores pre-post y tareas $[F(4,890)=2.71, p=.02]$ y las mediciones pre-post difirieron de manera significativa por el paso del tiempo $[F(1,890)=5.11, p=.02]$.

Se encontró que sólo la tarea de actividad de tiempo libre se modificó de manera significativa en las mediciones post, tanto en procedimientos temporales como en probabilísticos $[(t(91)=-3.54, p=.001)(t(91)=-2.60, p$ $=.011)]$ (ver Tabla 2).

Otras variables de análisis fueron la autoeficacia, evaluada con el cuestionario de confianza situacional (CCS); el índice subescala somatización; el índice subescala de depresión SCL-90; el índice subescala de ansiedad SCL-90; el índice subescala de psicoticismo; y el índice global de severidad GSDI. La variable CCS tiene un efecto principal de paso del tiempo $[F(1,53)=61.3$, $p$ $=.000]$, pero no por interacción con tipo de participante $[F(1,53)=3.56, p=.065]$; se observa un incremento en la variable en la medición post.

En el índice de la subescala de somatización se encontró un efecto de interacción entre tipo de participante $\mathrm{y}$ factor pre-post $[F(2,82)=4.72, p=.011]$. Las comparaciones planeadas con el test de Bonferroni indican que el cambio resultó significativo en los participantes en la categoría de tratamiento usual y autocontrol.

Para el índice de la subescala de depresión se encontró un efecto de interacción entre tipo de participante y factor pre-post $[F(2,82)=20.75, p=.000]$. Las comparaciones planeadas con el test de Bonferroni indican que el cambio resultó significativo en los participantes en la categoría de tratamiento usual y autocontrol.

Tabla 1

Características demográficas de los participantes $(n=92)$

\begin{tabular}{|c|c|c|c|c|}
\hline & $\begin{array}{c}\text { Control } \\
\mathrm{M}(\mathrm{SD}) \mathrm{O} \\
\%(\mathrm{~N})\end{array}$ & $\begin{array}{c}\text { Cocaína tx usual } \\
\mathrm{M}(\mathrm{SD}) \mathrm{O} \\
\%(\mathrm{~N})\end{array}$ & $\begin{array}{c}\text { Cocaína tx autocontrol } \\
\mathrm{M}(\mathrm{SD}) \mathrm{O} \\
\%(\mathrm{~N})\end{array}$ & $p^{a}$ \\
\hline N & 30 & 31 & 31 & \\
\hline Edad (en años) & $26.6(7)$ & $32.3(6.9)$ & $30.6(6.9)$ & $<.05$ \\
\hline Nivel educativo & & & & $<.01$ \\
\hline Secundaria & $0(0 \%)$ & $5(16.1 \%)$ & $8(25.8 \%)$ & \\
\hline Preparatoria & $17(56.6 \%)$ & 22 (70.9\%) & $16(51.6 \%)$ & \\
\hline Licenciatura & $13(43.3 \%)$ & $4(12.9 \%)$ & 7 (22.5\%) & \\
\hline Ingresos & & & & $>.01$ \\
\hline$<\$ 2500$ & $8(26.6 \%)$ & $4(12.9 \%)$ & 7 (22.5\%) & \\
\hline$\$ 2501-\$ 4000$ & $13(43.3 \%)$ & $17(54.8 \%)$ & $15(48.3 \%)$ & \\
\hline$>\$ 4001$ & $9(30 \%)$ & $10(32.2 \%)$ & $9(29 \%)$ & \\
\hline
\end{tabular}

a para evaluar si había diferencias entre los grupos se utilizaron los estadísticos $X^{2}$ y Fisher. 
Tabla 2

Evaluaciones de descuento antes y después del tratamiento

\begin{tabular}{|c|c|c|c|c|c|c|}
\hline & Control & & Tratamiento & Usual & Tratamiento & Autocontrol \\
\hline & M(SD) Pre & M(SD) Post & M(SD) Pre & M(SD) Post & M(SD) Pre & M(SD) Post \\
\hline $\begin{array}{l}\text { Bebida preferida } \\
\text { demorada }\end{array}$ & $.30(.26)$ & $.29(.27)$ & $.18(.21)$ & $.23(.21)$ & $.31(.34)$ & $.30(.32)$ \\
\hline $\begin{array}{l}\text { Actividad de tiempo } \\
\text { libre demorada }\end{array}$ & $.21(.20)$ & $.33(.27)^{\star}$ & $.17(.22)$ & $.23(.24)^{\star}$ & $.12(.13)$ & $.23(.26)^{\star}$ \\
\hline $\begin{array}{l}\text { Ganancia } \$ 200 \\
\text { demorada }\end{array}$ & $.34(.23)$ & $.32(.28)$ & $.16(.17)$ & $.23(.20)$ & $.33(.35)$ & $.24(.26)$ \\
\hline $\begin{array}{l}\text { Ganancia } \$ 3000 \\
\text { demorada }\end{array}$ & $.39(.32)$ & $.42(.28)$ & $.23(.24)$ & $.27(.28)$ & $.33(.37)$ & $.33(.34)$ \\
\hline $\begin{array}{l}\text { Pérdida } \$ 1500 \\
\text { demorada }\end{array}$ & $.56(.29)$ & $.52(.34)$ & $.50(.22)$ & $.45(.31)$ & $.39(.29)$ & $.41(.26)$ \\
\hline $\begin{array}{l}\text { Bebida preferida } \\
\text { probabilístico }\end{array}$ & $.31(.21)$ & $.31(.20)$ & $.23(.23)$ & $.28(.35)$ & $.17(.18)$ & $.26(.25)$ \\
\hline $\begin{array}{l}\text { Actividad de tiempo } \\
\text { libre probabilístico }\end{array}$ & $.26(.18)$ & $.29(.17)^{\star}$ & $.18(.16)$ & $.26(.20)^{\star}$ & $.18(.16)$ & $.27(.23)^{\star}$ \\
\hline $\begin{array}{l}\text { Ganancia } \$ 200 \\
\text { probabilístico }\end{array}$ & $.33(.22)$ & $.30(.22)$ & $.19(.15)$ & $.30(.25)$ & $.28(.25)$ & $.26(.21)$ \\
\hline $\begin{array}{l}\text { Ganancia } \$ 3000 \\
\text { probabilístico }\end{array}$ & $.21(.18)$ & $.28(.25)$ & $.26(.29)$ & $.30(.28)$ & $.25(.28)$ & $.22(.22)$ \\
\hline $\begin{array}{l}\text { Pérdida } \$ 1500 \\
\text { probabilístico }\end{array}$ & $.43(.25)$ & $.39(.25)$ & $.42(.27)$ & $.40(.26)$ & $.45(.28)$ & $.47(.24)$ \\
\hline
\end{tabular}

En el índice de la subescala de ansiedad se halló un efecto de interacción entre tipo de participante y factor pre-post $[F(2,82)=10.35, p=.000]$. Las comparaciones planeadas con el test de Bonferroni indican que el cambio resultó significativo únicamente en la categoría de tratamiento usual.

En el índice de la subescala de psicoticismo se encontró un efecto de interacción entre tipo de participante y factor pre-post $[F(2,82)=4.78, p=.011]$. Las comparaciones planeadas con el test de Bonferroni indican que el cambio resultó significativo en los participantes de las categorías de tratamiento usual y autocontrol.

En el índice GSI se encontró interacción entre el tipo de participante y el factor pre-post $[F(2,82)=9.28, p=$ .000]. Las comparaciones planeadas con el test de Bonferroni indican que el cambio resultó significativo en los participantes, en la categoría de tratamiento usual y autocontrol (ver Tabla 3).

La prueba Chi cuadrada $\left[X^{2}(2)=1.25, p=.53\right]$ arrojó que no hay diferencias significativas por tipo de tratamiento y número de participantes que recayeron. En

Tabla 3

Evaluaciones de CCS y SCL-90 antes y después del tratamiento

\begin{tabular}{|c|c|c|c|c|c|c|}
\hline \multicolumn{3}{|c|}{ Control } & \multirow{2}{*}{$\begin{array}{c}\text { Tratamiento } \\
\text { M(SD) } \\
\text { Pre }\end{array}$} & \multirow{2}{*}{$\begin{array}{c}\text { Usual } \\
\text { M(SD) } \\
\text { Post }\end{array}$} & \multirow{2}{*}{$\begin{array}{c}\text { Tratamiento } \\
\text { M(SD) } \\
\text { Pre }\end{array}$} & \multirow{2}{*}{$\begin{array}{c}\text { Autocontrol } \\
\mathrm{M}(\mathrm{SD}) \\
\text { Post }\end{array}$} \\
\hline & $\begin{array}{l}\text { M(SD) } \\
\text { Pre }\end{array}$ & $\begin{array}{l}\text { M(SD) } \\
\text { Post }\end{array}$ & & & & \\
\hline CCS & -------------- & -------------- & $54.1(23)$ & $83.3(15)^{\star}$ & $65.1(21)$ & $82.9(15)^{\star}$ \\
\hline Somatización & $.53(.59)$ & $.44(.62)$ & $.82(.67)$ & $.30(.27)^{\star}$ & $.49(.45)$ & $.29(.30)^{\star}$ \\
\hline Depresión & $.51(.47)$ & $.52(.53)$ & $1.22(.75)$ & $.34(.33)^{\star}$ & $.71(.62)$ & $.36(.42)^{\star}$ \\
\hline Ansiedad & $.33(.44)$ & $.40(.59)$ & $.75(.70)$ & $.26(.29)^{\star}$ & $.44(.42)$ & $.35(.57)$ \\
\hline Psicoticismo & $.26(.36)$ & $.22(.39)$ & $.53(.54)$ & $.21(.31)^{\star}$ & $.32(.39)$ & $.14(.21)^{*}$ \\
\hline $\begin{array}{l}\text { Severidad } \\
\text { global SCL-90 }\end{array}$ & $.42(.40)$ & $.43(.50)$ & $.51(.43)$ & $.32(.27)^{\star}$ & $.51(.43)$ & $.32(.27)^{\star}$ \\
\hline
\end{tabular}


el tratamiento usual, 12 participantes permanecieron en abstinencia, mientras que en autocontrol ocho permanecieron en abstinencia en la evaluación a tres meses.

No se encontraron diferencias significativas entre los que estaban en abstinencia y los que recayeron para las siguientes variables dependientes: AuC Post $[t(448)=$ -.236, $p=.814]$, CCS post [ $t(38)=-1.097, p=.279]$, somatización $[t(41)=-.055, p=.956]$, depresión post $[t(41)=-1.134, p=.263]$, ansiedad post $[t(41)=.937, p$ $=.354]$, psicoticismo $[t(41)=-.164, p=.870]$ y severidad global post $[t(41)=.070, p=.944]$.

La prueba Chi-cuadrada mostró $\left[X^{2}(1)=.007, p=\right.$ .93) que no hay diferencias significativas por tipo de tratamiento y número de participantes que no terminaron tratamiento.

No se encontraron diferencias significativas entre los participantes que terminaron y los que no terminaron tratamiento para cada una de las variables dependientes: AuC Pre $[t(1308)=-1.786, p=.074]$, CCS pre $[t(125)=-.282$, $p=.799]$, somatización [t(104) $=-1.25, p=.214]$, depresión pre $[t(104)=-.518, p=.605]$, ansiedad pre $[t(104)=$ -1.319, $p=.190]$, psicoticismo $[t(104)=-1.57, p=.119]$ y severidad global pre $[t(104)=-1.177, p=.242]$.

\section{DISCUSIÓN Y CONCLUSIONES}

El presente estudio tuvo como objetivo analizar los cambios en las tasas de descuento, auto-eficacia y sintomatología psicosomática en función de dos tipos de tratamiento psicológico y en ausencia de éste, en participantes con trastorno por uso de crack y en un grupo control sin trastorno por uso de drogas. Se observó que las tasas de descuento no se modifican con una intervención psicológica, aun en modalidades cognitivo-conductuales aplicadas al autocontrol, ya que el factor tipo de participante no resultó con interacción en los cambios después del tratamiento; este hallazgo apoya la literatura previa sobre la estabilidad de las tasas de descuento (Dennhardt et al., 2015; De Wilde et al., 2013; Jones, 2013; Landes et al., 2012; López, 2014; López-Torrecillas, Nieto-Ruiz et al., 2014; Secades-Villa et al., 2014; Weidberg et al., 2015; Weidel, 2013). Sin embargo, el presente estudio demostró la estabilidad en ambos procedimientos, temporales y probabilísticos, al considerar cuatro de las cinco tareas, ya que la de actividad de tiempo libre cambió en ambos procedimientos, sin interacción con el tipo de tratamiento. Este hallazgo resulta difícil de interpretar; no obstante, la variación en las diferentes actividades de preferencia que eligieron los participantes podría estar asociada con su inestabilidad en el tiempo.

Se sugiere que en futuras investigaciones se evalúe la frecuencia de realización de la actividad de preferencia elegida, esto con la intención de comparar si des- pués de una intervención psicológica se incrementa la realización de actividades recreativas que compitan con el uso de la droga.

De la misma manera, se buscó evaluar si altas tasas de descuento se asociaban con participantes que no terminaban el tratamiento en las evaluaciones previas; se observó que ninguna de las variables evaluadas difería significativamente entre pacientes que terminaban y abandonaban el tratamiento, lo cual contradice la literatura previa (López-Torrecillas, Perales et al., 2014). Al analizar las tasas de descuento en la post evaluación al tratamiento, éstas no diferían entre los participantes que recaían y los que se mantenían en abstinencia, lo cual apoya lo encontrado en investigaciones previas que sugieren que el descuento no es un buen predictor de la recaída (Jones, 2013; López-Torrecillas, Nieto-Ruiz et al., 2014; Passetti et al., 2011; Peters et al., 2013).

Estos hallazgos sugieren que en futuras investigaciones se aumente el tamaño de la muestra para realizar análisis de tiempo en un evento con una regresión de Cox, para evaluar el potencial predictivo del descuento con el tiempo de abstinencia. También se sugiere utilizar seguimientos a seis y 12 meses, para evaluar si una abstinencia sostenida hasta un año muestra cambios significativos en las tasas de descuento. La importancia de considerar estudios con seguimientos de mayor extensión de tiempo se vincula con la importancia de la disminución de los cambios neuronales desarrollados por el uso crónico de la sustancia, lo cual se podría relacionar con una disminución del descuento temporal pronunciado. Las investigaciones previas han encontrado que una abstinencia de drogas sostenida de varios meses contribuye a generar cambios morfológicos, establecidos previamente por la dependencia, en neuroimagen funcional de ciertas estructuras y en desempeño cognitivo (Chang \& Chronicle, 2007; Chang, Yakupov, Cloak, \& Ernst, 2006; Fuchs, Branham, \& See, 2006; Hollander \& Carelli, 2007; Lu, Grimm, Shaham, \& Hope, 2003). Por lo tanto, se recomienda también el monitoreo en seguimiento de variables conductuales como el patrón de consumo y las evaluaciones psicofisiológicas.

Otro análisis estadístico sugerido es el análisis de Growth Mixture Model, que puede permitir encontrar patrones de respuesta en estas tareas y ayudar a identificar, de acuerdo con este patrón, a aquellos participantes con mayor sensibilidad a la demora; de esta forma, se podrían dirigir los esfuerzos a encontrar cuáles son los mecanismos en los consumidores que permiten modificar estos patrones de respuesta.

Otro objetivo del estudio fue evaluar si otras variables, además del descuento, se modificaban con las intervenciones psicológicas. La autoeficacia es una variable 
que, contradictoriamente, en estudios previos ha mostrado incrementos después de intervenciones (Blevins, Stephens, Walker, \& Roffman, 2014; Connor et al., 2013; Hser, 2007; Wong \& Longshore, 2008; Worley et al., 2014) y estabilidad que indica que la variable no es predictora del tiempo en abstinencia (Fiorentine \& Hillhouse, 2003; Stein, Zane, \& Grella, 2012). Los participantes de este estudio se mostraron con mayor autoeficacia en las evaluaciones post, sin interacción con el tipo de tratamiento que se brindó. Sin embargo, esta variable no resultó ser diferente en mediciones post en aquellos participantes que recaían y en los que mantenían su abstinencia en el seguimiento a tres meses. Estos hallazgos corroboran que la prueba es sensible a los cambios por tratamiento, pero no se asocia con la predicción de recaída y el abandono del tratamiento.

Las subescalas del SCL-90 mostraron cambios significativos pre-post, que afectaron a los participantes usuarios de cocaína que recibieron tratamiento, lo cual no afectó a los participantes controles. Se evaluaron las subescalas de manera separada, lo cual indica que ambos tratamientos fueron eficaces en la reducción de síntomas asociados con el uso de drogas. Sólo la escala de ansiedad resultó interactuar con el tratamiento, lo cual indicaría que el tratamiento usual resultó más eficaz para disminuir esta sintomatología. No obstante, el hecho de encontrar cambios sólo en una subescala del SCL-90 no permite concluir que el tratamiento usual fue superior al entrenamiento de autocontrol; sin embargo, a pesar de no encontrar diferencias significativas entre los dos grupos de tratamiento usual y autocontrol en las mediciones pre, sí se observa que el grupo usual mostró niveles más altos de sintomatología en la escala, por lo que consideramos necesario que en futuras investigaciones se busque balancear los grupos de acuerdo con los puntajes pre en las pruebas psicológicas que se utilicen.

En algunas investigaciones previas, el SCL-90 ha resultado ser una prueba efectiva para evaluar cambios debido a tratamientos psicológicos y farmacológicos administrados para disminuir síntomas en las escalas de severidad global, depresión, hostilidad y sensibilidad interpersonal en usuarios de drogas (Jariani, Saaki, Nazari, \& Birjandi, 2010; López et al., 2008; Manzardo, Pendleton, Poje, Penick, \& Butler, 2015; Martinotti et al., 2010); ha demostrado, también, ser sensible al evaluar diferencias entre participantes que abandonan y terminan un tratamiento (Engel et al., 2015); y que, además, funciona como valor predictivo en algunas de sus subescalas (Engel et al., 2015). En contraste, en otras investigaciones la prueba no resultó ser sensible a cambios por tratamiento ni a diferencias entre los participantes que abandonan y los que terminan el tratamiento (Öhlin, Hesse, Fridell, \& Tätting, 2011); también se ha mostrado poco sensible a los efectos farmacológicos que drogas como el tabaco generan en los usuarios, en comparación con los no consumidores (López, Fernández, \& Becoña, 2009).

El presente estudio contribuye a la sensibilidad para evaluar cambios pre-post del SCL-90 debido al tratamiento; sin embargo, no corrobora hallazgos previos de su discriminación para participantes que abandonan tratamiento y participantes que recaen.

Los resultados de la presente investigación describen a la población masculina en concordancia con los resultados de la Encuesta Nacional de Adicciones 2011, que muestra que los incrementos significativos en el uso de cocaína se han dado en varones; adicionalmente, se consideró la baja tasa de mujeres que acuden a tratamientos residenciales por problemas relacionados con el uso de cocaína. Se sugiere que en futuras investigaciones se trabaje con mujeres para evaluar el efecto de género y descuento, lo cual ha sido ya indagado en previas investigaciones que han demostrado que el descuento pronunciado es característico de hombres (Myerson, Green, van den Berk-Clark, \& Grucza, 2015), en contraste con otros estudios que plantean la existencia de una mayor problemática asociada con el consumo de piedra en mujeres (Lejuez, Bornovalova, Reynolds, Daughters, \& Curtin, 2007); sin embargo, un metaanálisis de 227 estudios demostró que, en general, no se encuentran diferencias entre hombres y mujeres en tareas de descuento temporal y tareas de función ejecutiva (Cross, Copping, \& Campbell, 2011), por lo que futuros estudios en el tema son necesarios para clarificar el perfil de propensión al riesgo e impaciencia que presentan mujeres consumidoras, ya que el presente estudio sólo comprende el entendimiento del descuento en hombres consumidores.

El presente trabajo representó una investigación puente que permitió evaluar la utilidad clínica de las mediciones de descuento en usuarios que acuden a tratamiento para dejar el consumo de drogas. Se mostró que estas mediciones son un marcador fijo que no es susceptible de modificación como otras variables. Sin embargo, no se tuvo un control total en las intervenciones psicológicas y farmacológicas, por lo que se sugiere mayor rigurosidad en el control del tratamiento psicológico en futuras investigaciones, como utilizar diseños paralelos.

A pesar de sus limitaciones, este estudio provee evidencia sobre la validez externa de la estabilidad del descuento en situaciones no experimentales con variaciones contextuales en las tareas de elección, en las que se considere la abstinencia de los participantes en seguimiento; esto contribuye, a nivel teórico y práctico, a generar mejoras en el entendimiento de la conducta adictiva.

REVISTA INTERNACIONAL DE INVESTIGACIÓN EN ADICCIONES 2016.2(1).4-15 


\section{FUENTES DE FINANCIAMIENTO}

El presente proyecto fue financiado por Conacyt, beca número: 323655, y el proyecto PAPPIIT número: IN305114.

\section{CONFLICTOS DE INTERÉS}

Los autores declaran no tener conflictos de interés relacionados con el estudio o con los resultados presentados.

\section{AGRADECIMIENTOS}

Los autores extienden un especial agradecimiento a Centros de Integración Juvenil, A.C. y al Instituto de Asistencia e Integración Social del gobierno del Distrito Federal por su apoyo para la implementación del estudio. De la misma forma, agradecemos a los doctores Leonard Green y Joel Myerson, de la Universidad de Washington, en Saint Louis Missouri, por su asesoramiento metodológico y teórico.

\section{REFERENCIAS}

American Psychiatric Association. (2013). Diagnostic and statistical manual of mental disorders (DSM-5). Washington: American Psychiatric Association.

Barragán, L., Flores, M., Medina-Mora, M. E., \& Ayala, H. (2007). Modelo integral de satisfacción cotidiana en usuarios dependientes de alcohol y otras drogas. Salud Mental, 30(3), 29-38.

Bickel, W. K., Yi, R., Landes, R. D., Hill, P. F., \& Baxter, C. (2011). Remember the future: working memory training decreases delay discounting among stimulant addicts. Biological Psychiatry, 69(3), 260-265. doi: 10.1016/j.biopsych.2010.08.017

Black, A. C., \& Rosen, M. (2011). A money management-based substance use treatment increases valuation of future rewards. Addictive Behaviors, 36(1), 125-128. doi: 10.1016/j.addbeh.2010.08.014

Blevins, C. E., Stephens, R. S., Walker, D. D., \& Roffman, R. A. (2014). Situational determinants of use and treatment outcomes in marijuana dependent adults. Addictive Behaviors, 39(3), 546-552. doi: 10.1016/j.addbeh.2013.10.031

Chang, L., \& Chronicle, E. P. (2007). Functional imaging studies in cannabis users. The Neuroscientist, 13(5), 422-432. doi: 10.1177/1073858406296601

Chang, L., Yakupov, R., Cloak, C., \& Ernst, T. (2006). Marijuana use is associated with a reorganized visual-attention network and cerebellar hypoactivation. Brain, 129(5), 1096-1112. doi: 10.1093/brain/ awl064

Coffey, S. F., Gudleski, G. D., Saladin, M. E., \& Brady, K. T. (2003). Impulsivity and rapid discounting of delayed hypothetical rewards in cocaine-dependent individuals. Experimental and Clinical Psychopharmacology, 11(1), 18-25. doi: 10.1037/1064-1297.11.1.18

Connor, J., Gullo, M., Chan, G., Young, R., Hall, W., \& Feeney, G. (2013). Polysubstance use in cannabis users referred for treatment: drug use profiles, psychiatric comorbidity and cannabis-related beliefs. Frontiers in Psychiatry, 4(79), 1-7. doi: 10.3389/fpsyt.2013.00079

Cross, C. P., Copping, L. T., \& Campbell, A. (2011). Sex differences in impulsivity: a meta-analysis. Psychological Bulletin, 137(1), 97-130. doi: 10.1037/a0021591

Cruz, C., López, L., Blas, C., González, L., \& Chávez, R. (2004). Datos sobre la validez y confiabilidad de la Symptom Check List 90 (SCL-90) en una muestra de sujetos mexicanos. Salud Mental, 28(1), 72-81.

Dallery, J., \& Raiff, B. (2007). Delay discounting predicts cigarette smoking in a laboratory model of abstinence reinforcement. Psychopharmacology, 190(4), 485-496. doi: 10.1007/s00213006-0627-5

Dennhardt, A. A., Yurasek, A. M., \& Murphy, J. G. (2015). Change in delay discounting and substance reward value following a brief alcohol and drug use intervention. Journal of the Experimental Analysis of Behavior, 103(1), 125-140. doi: 10.1002/jeab.121

De Wilde, B., Bechara, A., Sabbe, B., Hulstijn, W., \& Dom, G. (2013). Risky decision-making but not delay discounting improves during inpatient treatment of polysubstance dependent alcoholics. Frontiers in Psychiatry, 4(91), 1-7. doi: 10.3389/fpsyt.2013.00091

Echeverría, L., \& Ayala, H. (1977). Cuestionario de confianza situacional: traducción y adaptación. México: Universidad Nacional Autónoma de México.

Engel, K., Schaefer, M., Stickel, A., Binder, H., Heinz, A., \& Richter, C. (2015). The role of psychological distress in relapse prevention of alcohol addiction. Can high scores on the SCL-90-R predict alcohol relapse? Alcohol and Alcoholism, 51(1), 27-31. doi: 10.1093/alcalc/agv062

Epstein, L., \& Roemmich, J. (2001). Reducing sedentary behavior: Role in modifying physical activity. Exercise \& Sports Sciences Reviews, 29(3), 103-108. doi: 0091-6631/2903/103-108

Fiorentine, R., \& Hillhouse, M. P. (2003). Why extensive participation in treatment and twelve-step programs is associated with the cessation of addictive behaviors: an application of the addicted-self model of recovery. Journal of Addictive Diseases, 22(1), 35-55. doi: 10.1080/10550490390226932

Fuchs, R. A., Branham, R. K., \& See, R. E. (2006). Different neural substrates mediate cocaine seeking after abstinence versus extinction training: a critical role for the dorsolateral caudateputamen. The Journal of Neuroscience, 26(13), 3584-3588. doi: 10.1523/JNEUROSCI.5146-05.2006

García-Rodríguez, O., Secades-Villa, R., Weidberg, S., \& Yoon, J. (2013). A systematic assessment of delay discounting in relation to cocaine and nicotine dependence. Behavioral Processes, 99 100-105. doi:10.1016/j.beproc.2013.07.007

Heil, S., Johnson, M., Higgins, S., \& Bickel, W. (2006). Delay discounting in currently using and currently abstinent cocaine-dependent outpatients and non-drug-using matched controls. Addictive Behaviors, 31(7), 1290-1294. doi:10.1016/j.addbeh.2005.09.005

Hollander, J. A., \& Carelli, R. M. (2007). Cocaine-associated stimuli increase cocaine seeking and activate accumbens core neurons after abstinence. The Journal of Neuroscience, 27(13), 
3535-3539. doi: 10.1523/JNEUROSCI.3667-06.2007

Holt, D., Green, L., \& Myerson, J. (2012). Estimating the subjective value of future rewards: comparison of adjusting amount and adjusting delay procedures. Behavioural Processes, 90(3), 302310. doi:10.1016/j.beproc.2012.03.003

Hser, Y. I. (2007). Predicting long-term stable recovery from heroin addiction: Findings from a 33-year follow-up study. Journal of Addictive Diseases, 26(1), 51-60. doi: 10.1300/J069v26n01_07

Jariani, M., Saaki, M., Nazari, H., \& Birjandi, M. (2010). The effect of Olanzapine and Sertraline on personality disorder in patients with methadone maintenance therapy. Psychiatria Danubina, 22(4), 544-547.

Jones, C. (2013). Delay discounting rates, relapse, and treatment satisfaction in young adults. (Doctoral dissertation). Minnesota: Capella University. http://search.proquest.com/docview/ 1492338109

Kazdin, A. E. (2011). Single-case research designs: Methods for clinical and applied settings. Oxford: Oxford University Press.

Krishnan-Sarin, S., Reynolds, B., Duhig, A. M., Smith, A., Liss, T., McFetridge, A., ... Potenza, M. N. (2007). Behavioral impulsivity predicts treatment outcome in a smoking cessation program for adolescent smokers. Drug and Alcohol Dependence, 88(1), 79-82. doi:10.1016/j.drugalcdep.2006.09.006

Landes, R. D., Christensen, D. R., \& Bickel, W. K. (2012). Delay discounting decreases in those completing treatment for opioid dependence. Experimental and Clinical Psychopharmacology, 20(4), 302-309. doi: 10.1037/a0027391

Lejuez, C. W., Bornovalova, M. A., Reynolds, E. K., Daughters, S. B., \& Curtin, J. J. (2007). Risk factors in the relationship between gender and crack/cocaine. Experimental and Clinical Psychopharmacology, 15(2), 165-175. doi: 10.1037/1064-1297.15.2.165

Linage, L., \& Lucio, E. (2013). Propiedades psicométricas del ASSIST en una muestra de estudiantes mexicanos. Revista Española de Drogodependencias, 38(1), 37-51.

López, A. A. (2014). Examining delay discounting and response to incentive-based smoking-cessation treatment among pregnant women. (Doctoral dissertation). Estados Unidos: The University of Vermont.

López, A., Becoña, E., Vieitez, I., Cancelo, J., Sobradelo, J., García, J. M., ... Lage, M. T. (2008). ¿Qué ocurre a los 24 meses del inicio del tratamiento en una muestra de personas con dependencia de la cocaína? Adicciones, 20(4), 347-356. doi: 10.20882/adicciones.258

López, A., Fernández, E., \& Becoña, E. (2009). Comparación de las puntuaciones del SCL-90-R entre personas con dependencia de la nicotina y personas con dependencia de la cocaína al inicio del tratamiento. Revista de Psicopatología y Psicología Clínica, 14(1), 17-23. doi: 10.5944/rppc.vol.14.num.1.2009.4063

López-Torrecillas, F., Nieto-Ruiz, A., Velasco-Ortuño, S., Lara-Fernández, M., López-Quirantes, E. M., \& Castillo-Fernández, E. (2014). The role of impulsivity in dropout from treatment for cigarette smoking. Comprehensive Psychiatry, 55(7), 1609-1613. doi:10.1016/..comppsych.2014.06.004

López-Torrecillas, F., Perales, J. C., Nieto-Ruiz, A., \& Verdejo-Gar- cía, A. (2014). Temperament and impulsivity predictors of smoking cessation outcomes. PLOS ONE, 9(12), 1-18. doi:10.1371/ journal.pone.0112440

Lu, L., Grimm, J. W., Shaham, Y., \& Hope, B. T. (2003). Molecular neuroadaptations in the accumbens and ventral tegmental area during the first 90 days of forced abstinence from cocaine selfadministration in rats. Journal of Neurochemistry, 85(6), 16041613. doi: 0.1046/j.1471-4159.2003.01824.x

MacKillop, J., \& Kahler, C. W. (2009). Delayed reward discounting predicts treatment response for heavy drinkers receiving smoking cessation treatment. Drug and Alcohol Dependence, 104(3), 197-203. doi:10.1016/j.drugalcdep.2009.04.020

Manzardo, A. M., Pendleton, T., Poje, A., Penick, E. C., \& Butler, M. G. (2015). Change in psychiatric symptomatology after benfotiamine treatment in males is related to lifetime alcoholism severity. Drug and Alcohol Dependence, 152, 257-263. doi: 10.1016/j. drugalcdep.2015.03.032

Manwaring, J., Green, L., Myerson, J., Strube, M., \& Wilfley, D. (2011). Discounting of various types of rewards by women with and without binge eating disorder: evidence for general rather than specific differences. The Psychological Record, 61(4), 561-582.

Marín-Navarrete, R., Templos-Nuñez, L., Eliosa-Hernández, A., ViIlalobos-Gallegos, L., Fernández-Mondragón, J., Pérez-López, A., ... Horigian, V. E. (2014). Characteristics of a treatment-seeking population in outpatient addiction treatment centers in Mexico. Substance Use \& Misuse, 49(13), 1784-1794. doi: 10.3109/10826084.2014.931972

Martinotti, G., Di Nicola, M., Frustaci, A., Romanelli, R., Tedeschi, D., Guglielmo, R., ... Di Giannantonio, M. (2010). Pregabalin, tiapride and lorazepam in alcohol withdrawal syndrome: a multi-centre, randomized, single-blind comparison trial. Addiction, 105(2), 288-299. doi: 10.1111/j.1360-0443.2009.02792.x

Mejía, D. (2012). Efectividad de una intervención grupal para la dependencia al alcohol en pacientes con diagnostico dual reporte de experiencia profesional. (Tesis de Maestría). Ciudad de México: Facultad de Psicología, UNAM.

Mejía, D., Morales, S., \& Nieto, J. (2015). Descuento temporal asociado al uso de cocaína. Revista Mexicana de Análisis de la Conducta, 41(1), 86-101.

Morrison, K., Madden, G., Odum, A., Friedel, J., \& Twohing, M. (2014). Altering impulsive decision making with an acceptance-based procedure. Behavior Therapy, 45(5), 630-639. doi:10.1016/j.beth.2014.01.001

Myerson, J., Green, L., van den Berk-Clark, C., \& Grucza, R. A. (2015). Male, but not female, alcohol-dependent African Americans discount delayed gains more steeply than propensity-score matched controls. Psychopharmacology, 232(24), 44934503. doi: 10.1007/s00213-015-4076-x

Myerson, J., Green, L., \& Warusawitharana, M. (2001). Area under the curve as a measure of discounting. Journal of the Experimental Analysis of Behavior, 76(2), 236-243. doi: 10.1901/ jeab.2001.76-235

Öhlin, L., Hesse, M., Fridell, M., \& Tätting, P. (2011). Poly-substance 
use and antisocial personality traits at admission predict cumulative retention in a buprenorphine programme with mandatory work and high compliance profile. BMC Psychiatry, 11(1), 1-8. doi:10.1186/1471-244X-11-81

Ohmura, Y., Takahashi, T., Kitamura, N., \& Wehr, P. (2006). Three-month stability of delay and probability discounting measures. Experimental and Clinical Psychopharmacology, 14(3), 318-328. doi: 10.1037/1064-1297.14.3.318

Passetti, F., Clark, L., Davis, P., Mehta, M. A., White, S., Checinski, K., ... Abou-Saleh, M. (2011). Risky decision-making predicts short-term outcome of community but not residential treatment for opiate addiction. Implications for case management. Drug and Alcohol Dependence, 118(1), 12-18. doi:10.1016/j.drugalcdep.2011.02.015

Peters, E. N., Petry, N. M., LaPaglia, D. M., Reynolds, B., \& Carroll, K. M. (2013). Delay discounting in adults receiving treatment for marijuana dependence. Experimental and Clinical Psychopharmacology, 21(1), 46-54. doi: 10.1037/a0030943

Secades-Villa, R., Weidberg, S., García-Rodríguez, O., Fernández-Hermida, J. R., \& Yoon, J. H. (2014). Decreased delay discounting in former cigarette smokers at one year after treatment. Addictive Behaviors, 39(6), 1087-1093. doi:10.1016/j. addbeh.2014.03.015

Secretaría de Salud, Centro Nacional para la Prevención y el Control de las Adicciones, Comisión Nacional contra las Adicciones, Instituto Nacional de Psiquiatría Ramón de la Fuente Muñiz, Instituto Nacional de Salud Pública. (2012). Encuesta Nacional de Adicciones 2011. México: Autor. Recuperado de http://www.conadic.salud.gob.mx/pdfs/ENA_2011_DROGAS_ILICITAS_.pdf

Sheehan, D., Lecrubier, Y., \& Sheehan, K. (1998). The Mini-International Neuropsychiatric Interview (MINI): The development and validation of a structured diagnostic psychiatric interview for DSM-IV and ICD-10. Journal of Clinical Psychiatry, 59(20), 22-33.

Sheffer, C., MacKillop, J., McGeary, J., Landes, R., Carter, L., Yi, R., ... Bickel, W. (2012). Delay discounting, locus of control, and cognitive impulsiveness independently predict tobacco dependence treatment outcomes in a highly dependent, lower socioeconomic group of smokers. The American Journal on Addictions, 21(3), 221-232. doi: 10.1111/j.1521-0391.2012.00224.x

Skinner, H. (1982). The Drug Abuse Screening Test. Addictive Behaviors, 7(4), 363-371. doi: 10.1016/0306-4603(82)90005-3

Stanger, C., Ryan, S. R., Fu, H., Landes, R. D., Jones, B. A., Bickel, W. K., ... Budney, A.J. (2012). Delay discounting predicts adolescent substance abuse treatment outcome. Experimental and Clinical Psychopharmacology, 20(3), 205-212. doi:10.1037/ a0026543.

Stein, J. A., Zane, J. I., \& Grella, C. E. (2012). Impact of abstinence self-efficacy and treatment services on physical health-related behaviors and problems among dually diagnosed patients. Journal of Dual Diagnosis, 8(1), 64-73. doi: 10.1080/15504263.2012.647470

Stevens, L., Verdejo-García, A., Roeyers, H., Goudriaan, A. E., \&
Vanderplasschen, W. (2015). Delay discounting, treatment motivation and treatment retention among substance-dependent individuals attending an in inpatient detoxification program. Journal of Substance Abuse Treatment, 49, 58-64. doi:10.1016/j. jsat.2014.08.007

Tulsky, D., \& Zhu, J. (2003). Escala de Inteligencia Wechsler para adultos III: Manual técnico. México: Manual moderno.

Villalobos-Gallegos, L., Pérez-López, A., Mendoza-Hassey, R., Graue-Moreno, J., \& Marín-Navarrete, R. (2015). Psychometric and diagnostic properties of the Drug Abuse Screening Test (DAST): Comparing the DAST-20 vs. the DAST-10. Salud Mental, 38(2), 89-94.

Washio, Y., Higgins, S. T., Heil, S. H., McKerchar, T. L., Badger, G. J., Skelly, J. M., ... Dantona, R.L. (2011). Delay discounting is associated with treatment response among cocaine-dependent outpatients. Experimental and Clinical Psychopharmacology, 19(3), 243-248. doi: 10.1037/a0023617

Weidberg, S., García-Rodríguez, O., Yoon, J. H., \& Secades-Villa, R. (2015). Interaction of depressive symptoms and smoking abstinence on delay discounting rates. Psychology of Addictive Behaviors, 29(4), 1041-1047. doi: 10.1037/adb0000073

Weidberg, S., Landes, R. D., García-Rodríguez, O., Yoon, J. H., \& Secades-Villa, R. (2015). Interaction effect of contingency management and sex on delay-discounting changes among treatment-seeking smokers. Experimental and Clinical Psychopharmacology, 23(5), 361-368. doi: 10.1037/pha0000043

Weidel, J. J. (2013). The relationship of temporal discounting and working alliance to substance abuse treatment process in hispanic adolescents. (Doctoral dissertation). Florida, Estados Unidos: The University of Miami. http://scholarlyrepository.miami. edu/oa_dissertations/1088

WHO ASSIST Working Group. (2002). The Alcohol, Smoking and Substance Involvement Screening Test (ASSIST): development, reliability and feasibility. Addiction, 97(9), 1183-1194. doi: 10.1046/j.1360-0443.2002.00185.x

Wong, E. C., \& Longshore, D. (2008). Ethnic identity, spirituality, and self-efficacy influences on treatment outcomes among Hispanic American methadone maintenance clients. Journal of Ethnicity in Substance Abuse, 7(3), 328-340. doi: 10.1080/ 15332640802313478

Worley, M. J., Trim, R. S., Tate, S. R., Roesch, S. C., Myers, M. G., \& Brown, S. A. (2014). Self-efficacy and social networks after treatment for alcohol or drug dependence and major depression: Disentangling person and time-level effects. Psychology of Addictive Behaviors, 28(4), 1220- 1229. doi: 10.1037/ a0037901

Yi, R., Johnson, M. W., Giordano, L. A., Landes, R. D., Badger, G. J., \& Bickel, W. K. (2008). The effects of reduced cigarette smoking on discounting future rewards: an initial evaluation. The Psychological Record, 58(2), 163-174.

Yi, R., \& Landes, R. D. (2012). Temporal and probability discounting by cigarette smokers following acute smoking abstinence. Nicotine \& Tobacco Research, 14(5), 547-558. doi: 10.1093/ntr/ntr252 